IdeAs

Idées d'Amériques

16 | 2020

Les marges créatrices : intellectuel.le.s afrodescendant.e.s et indigènes auX Amériques, XIX-XXe siècle

\title{
Introduction. Le centenaire du 19e amendement aux Etats-Unis : enjeux politiques et historiographiques
}

\section{Hélène Quanquin}

\section{OpenEdition}

\section{Journals}

\section{Édition électronique}

URL : http://journals.openedition.org/ideas/9423

DOI : $10.4000 /$ ideas.9423

ISSN : 1950-570

\section{Éditeur}

Institut des Amériques

\section{Référence électronique}

Hélène Quanquin, «Introduction. Le centenaire du 19e amendement aux Etats-Unis : enjeux politiques et historiographiques », IdeAs [En ligne], 16 | 2020, mis en ligne le 01 octobre 2020, consulté le 18 octobre 2020. URL : http://journals.openedition.org/ideas/9423 ; DOI : https://doi.org/10.4000/ideas. 9423

Ce document a été généré automatiquement le 18 octobre 2020

\section{c)}

IdeAs - Idées d'Amériques est mis à disposition selon les termes de la licence Creative Commons Attribution - Pas d'Utilisation Commerciale - Pas de Modification 4.0 International. 


\title{
Introduction. Le centenaire du 19e amendement aux Etats-Unis : enjeux politiques et historiographiques
}

\author{
Hélène Quanquin
}

1 Le 18 août 1920 , le Tennessee devient le $36^{\mathrm{e}}$ Etat à ratifier le $19^{\mathrm{e}}$ amendement qui élimine toute limite du droit de vote fondée sur le genre. Le 26 août, cet amendement est officiellement intégré à la Constitution des Etats-Unis. Le $100^{\mathrm{e}}$ anniversaire de son adoption résonne de plusieurs façons avec le contexte actuel. Allison $\mathrm{K}$. Lange montre ainsi que l'épidémie de grippe ralentit le processus de ratification et força les suffragistes à revoir leurs stratégies, écho de la pandémie actuelle. Les femmes sont également au centre des élections à venir. La nomination de Kamala Harris comme colistière de Joe Biden met en évidence la longue histoire de l'engagement politique des femmes noires notamment au sein du Parti démocrate. Le décès récent de la juge à la Cour Suprême Ruth Bader Ginsburg pose enfin la question de la menace qui pèse de façon récurrente sur le droit à l'avortement aux Etats-Unis.

2 Les commémorations du $100^{\mathrm{e}}$ anniversaire $\mathrm{du} 19^{\mathrm{e}}$ amendement ont pris plusieurs formes: des événements officiels organisés par la Women's Suffrage Centennial Commission mise en place par le Congrès en 2017 ; des expositions physiques et en ligne ${ }^{1}$ ; l'érection de monuments et statues ; mais aussi la publication d'ouvrages importants nous invitant à revoir l'histoire du suffrage féminin aux Etats-Unis². Le centenaire a ainsi été l'occasion de remettre en cause plusieurs récits dominants touchant à l'histoire des mobilisations et des droits.

3 Le premier mythe est que le $19^{\mathrm{e}}$ amendement aurait donné le droit de vote à toutes les Américaines et institué le suffrage universel. Or, l'histoire du suffrage aux Etats-Unis est tout sauf linéaire; elle est au contraire faite de progrès et de régressions qui sont le résultat de mobilisations progressistes mais aussi conservatrices (Keyssar A., 2000). Certaines femmes avaient ainsi le droit de vote dans certains Etats et territoires avant 1920. Cela explique que la première femme élue au Congrès, Jeannette Rankin, l'ait été en 1916 puisque son Etat, le Montana, avait voté un amendement sur le suffrage deux 
ans auparavant. De façon encore plus significative, de nombreuses personnes continuèrent à être exclues du vote après 1920 : certaines Américaines ayant épousé un étranger jusque dans les années 1940 ; des Amérindiennes et Amérindiens avant les années 1960 ; les Noir.e.s dans le Sud mais aussi le Nord, victimes de lois et pratiques les privant de leurs droits. En 1965, le Voting Rights Act, voté sous la pression des mobilisations pour les droits civiques, déclara toute restriction du droit de vote illégale et imposa un contrôle fédéral à certains Etats. Aujourd'hui encore, les pratiques dites de voter suppression continuent de limiter l'exercice du suffrage (Anderson C., 2018).

Les commémorations du $19^{\mathrm{e}}$ amendement ont également remis en cause le récit d'un mouvement suffragiste composé en grande partie de femmes blanches de la classe moyenne. Les femmes racisées ont été une force importante du combat, malgré leur exclusion par les militantes blanches. La contribution de Sarah Gordon montre que cette invisibilisation à l'époque s'est doublée d'une invisibilisation dans les récits officiels et jusque dans les archives que plusieurs expositions et ouvrages récents se sont attachés à remettre en cause. La polémique autour du Women's Rights Pioneers Monument, la statue érigée à Central Park pour célébrer le combat suffragiste, est un bon exemple de ce phénomène. Le concept du monument, à l'origine centré sur deux suffragistes blanches fut modifié à de nombreuses reprises afin d'inclure une militante noire, Sojourner Truth (McGreevy N., 2020).

Le troisième et dernier mythe sur lequel le centenaire $\mathrm{du} 19^{\mathrm{e}}$ amendement nous permet de revenir est l'idée que le droit de vote aurait été une fin en soi pour les féministes. L'histoire du suffrage féminin aux Etats-Unis montre que celui-ci ne peut pas être pensé indépendamment de l'égalité générale des droits. Cette conviction, partagée dès les années 1920 par de nombreuses militantes, a nourri jusqu'à nos jours le combat pour l' Equal Rights Amendment étudié par Claire Delahaye. De la même façon, Jeanne Boiteux démontre que nombre d'Américaines ont toujours pensé le droit de vote en lien avec la citoyenneté économique et sociale.

Suffrage during the Pandemics of 1918 and 2020 - Allison K. Lange

Créer des expositions sur le suffrage, un enjeu et un plaisir - Sarah Anne Gordon

Citoyenneté économique et citoyenneté politique des femmes aux Etats-Unis - Jeanne Boiteux

Inscrire l'égalité des sexes dans la Constitution étatsunienne: Equal Rights Amendment, promesses et déboires d'une campagne interminée - Claire Delahaye

\section{BIBLIOGRAPHIE}

Anderson, Carol, One Person, No Vote : How Voter Suppression Is Destroying Our Democracy, Bloomsbury, 2018.

Keyssar, Alexander, The Right to Vote: The Contested History of Democracy in the United States, New York, Basic Books, 2000.

McGreevy, Nora, « Why the First Monument of Real Women in Central Park Matters - and Why It's Controversial », Smithsonian Magazine, 26 août 2020. https://www.smithsonianmag.com/ 
smart-news/monument-controversy-women-pioneer-central-park-180975662/ Consulté le 20 septembre 2020 .

\section{NOTES}

1. Voir à titre d'exemples les expositions suivantes: Hotbed, New-York Historical Society, 3 novembre 2017-25 mars 2018; Votes for Women : A Portrait of Persistence, National Portrait Gallery, Washington, D.C., 29 mars 2019-5 janvier 2020 ; «Can She Do It? » Massachusetts Debates a Woman's Right to Vote, Massachusetts Historical Society, 26 avril 2019-21 septembre 2019; Rightfully Hers: American Women and the Vote, National Archives Museum, Washington, D.C., 10 mai 2019-3 janvier 2021 ; Shall Not Be Denied: Women Fight For the Vote, Bibliothèque du Congrès, juin 2019-septembre 2020 ; Women March, New-York Historical Society, 28 février 2020-24 janvier 2021. Voir également les expositions en ligne: Truth Be Told: Stories of Black Women's Fight For the Vote; Seeing Citizens: Picturing American Women's Fight for the Vote, Radcliffe Institute for Advanced Study, Universite de Harvard.

2. Voir par exemple les ouvrages récents ou à venir: Cathleen Cahill, Recasting the Vote: How Women of Color Transformed the Suffrage Movement, Chapel Hill, NC: The University of North Carolina Press, 2020; Martha S. Jones, Vanguard: How Black Women Broke Barriers, Won the Vote, and Insisted on Equality for All, New York: Basic Books, 2020; Allison K. Lange, Picturing Political Power: Images in the Women's Suffrage Movement, Chicago, The University of Chicago Press, 2020; Kate Clarke Lemay, dir., Votes for Women: A Portrait of Persistence, Princeton, Princeton University Press, 2019 ; Alison S. Parker, Unceasing Militant: The Life of Mary Church Terrell, Chapel Hill, NC: The University of North Carolina Press, 2020.

\section{AUTEUR}

\section{HÉLÈNE QUANQUIN}

Université de Lille 